\title{
Distortion and Unbalance Assessing Indices Using Commercial Analysers
}

\author{
P. Salmerón ${ }^{1}$, Reyes S. Herrera ${ }^{2}$, A. Pérez ${ }^{3}$ and J. Prieto ${ }^{4}$ \\ Department of Electrical Engineering, E.P.S., Huelva University \\ Campus of La Rábida, Ctra.Palos de la Frontera s/n, 21819 Palos de la Frontera, Huelva (Spain) \\ phone:+34 959 017572, fax:+34 959017304 , \\ e-mail:1 patricio@uhu.es, ${ }^{2}$ reyes.sanchez@die.uhu.es, ${ }^{3}$ aperez@uhu.es, ${ }^{4}$ jpthomas@uhu.es
}

\begin{abstract}
Power quality monitoring is assuming an increasing importance because of the large diffusion of nonlinear and time-varying single-phase and three-phase loads and their interactions with the power supply system. This has made that the industrial electrical consumer systematically uses measure equipment to evaluate the electric power quality. The measurements show that the harmonics of the waveforms are variable along with the time due to the changes in the load conditions and in the system configuration. Thus, the electrical users dispose of a great quantity of data from such of the three phases of the supply. Therefore, it is necessary to find a way to characterize this great group of registered data. Thus, the objectives of this work are: firstly, to propose two indices, a three-phase total demand distortion index and a three-phase total unbalance index; secondly, this paper proposes a simple way to characterize the groups of data in an statistical way which make easy its representation. The study carried out is applied to the data recently registered in an electrical power system.
\end{abstract}

Key words: Electric power quality, evaluation index, harmonics.

\section{Introduction}

In recent years, power-quality issues have captured the attention of many researches and they have been the subject of numerous papers published in technical literature. The problem of controlling power quality is becoming more relevant because of the widespread use of nonlinear and time-varying single-phase or three-phase loads that increasingly affect the operation of distribution networks in residential, commercial, and industrial areas.

Power-quality deterioration is due to transient disturbances (voltage sags, voltage swells, impulses, etc.) and steady state disturbances (harmonic distortion, unbalance, flicker). This paper is focused on the second group, and, specifically on harmonic and unbalance phenomena.

The presence of harmonics in electrical networks poses many problems to power systems engineers. Their effects on power systems devices include resonance, reduced operating life of rotating machines, malfunctioning of power system protection devices, errors in power measurements, additional losses, etc.
Unbalance phenomena should also be well monitored, detected, and corrected because of the problems that they can generate. For example, a machine operating under an unbalance supply will draw a current with a degree of unbalance several times that of the supply voltage. As a consequence, each phase current might have very different rms values and the machine temperature would hardly rises. In fact, the more expensive motors and generators use to be fitted with appropriate protection to detect extreme unbalance.

The quality problems have supposed, from the user side, the necessary use of measure equipment such as electric power quality analysers with the target of monitoring the installation, [1-4]. This equipment disposes of a great number of quantities related to the harmonic distortion for each phase. Each measurement is composed by the voltage and current total harmonic distortion index (THD), their rms value and their fundamental component amplitude. The problem of these data characterization is the harmonic distortion representation when the installation has very different distortion levels in each phase. There are two possibilities. The first one is to consider each phase distortion level in a separate way; it supposes to treat three times more information. The other possibility is to characterize the installation distortion through a global parameter which considers the whole three-phase system. This is the approach assumed in this paper. Besides, taking into account that the system voltage and current waveforms vary along with the time, the registered data suffer incertitude with the different measure times. Due to that, the data behave in a random way and they need to be descript in a statistical form, [5-8].

In this paper, in the second section, they are defined the more appropriated distortion and unbalance indices applied to three-phase four-wire networks, based on commercial analysers measurements. In the third section it is shown the voltage and current waveforms variation along with the time and the convenience of using statistical techniques to characterize the data. Finally, in the fourth section they are introduced some total demand weighted distortion and unbalance indices to evaluate the power quality from the measures got with commercial 
analysers. The proposed indices have been applied to a group of data registered in an installation with fifteen minutes intervals.

\section{Power quality indices}

For a three-phase four-wire system it is defined the equivalent three-phase voltage $\mathrm{V}_{\mathrm{e}}$ as follows, [9]:

$$
V_{e}=\sqrt{\frac{V_{L 1}^{2}+V_{L 2}^{2}+V_{L 3}^{2}+V_{L 4}^{2}}{3}}
$$

where $\mathrm{V}_{\mathrm{Lj}}$ is the voltage rms value of each wire respect to an artificial neutral which makes that those voltages sum is null. For this reason, the numerator fourth term may be neglected and the expression (1) may be approximated by the next:

$$
V_{e}=\sqrt{\frac{V_{L 1}^{2}+V_{L 2}^{2}+V_{L 3}^{2}}{3}}
$$

In another way, the equivalent three-phase current $\mathrm{I}_{\mathrm{e}}$ may be defined as follows:

$$
I_{e}=\sqrt{\frac{I_{L 1}^{2}+I_{L 2}^{2}+I_{L 3}^{2}+I_{L 4}^{2}}{3}}
$$

Traditionally, the distortion have been characterized by the total harmonic distortion indices (directly measured by the analyser) defined as follows:

$$
\begin{aligned}
& V T H D_{1 \phi}=\sqrt{\frac{V_{L 1}^{2}-V_{L 11}^{2}}{V_{L 11}^{2}}} \\
& \text { ITHD }_{1 \phi}=\sqrt{\frac{I_{L 1}^{2}-I_{L 11}^{2}}{I_{L 11}^{2}}}
\end{aligned}
$$

where $V_{L 1}$ and $I_{L 1}$ are each phase voltage and current rms value, respectively, and $\mathrm{V}_{\mathrm{L} 11}$ and $\mathrm{I}_{\mathrm{L} 11}$ their fundamental component amplitude.

Another way of controlling the harmonic content is through the total demand distortion index (TDD) defined as follows:

$$
\begin{aligned}
& V T D D_{1 \phi}=\sqrt{\frac{V_{L 1}^{2}-V_{L 11}^{2}}{V_{L 1}^{2}}} \\
& I T D D_{1 \phi}=\sqrt{\frac{I_{L 1}^{2}-I_{L 11}^{2}}{I_{L 1}^{2}}}
\end{aligned}
$$

These indices are similar to the common THD. In one hand, THD relates the waveform harmonic part to its fundamental component. On the other hand, TDD relates that same harmonic part to the waveform rms value. In this way, they are avoided the problems derived from an index which would be infinite in the case of waveforms with a null fundamental component.

Taking into account both equivalent three-phase rms values presented in (2) and (3), it is possible to characterize the three-phase system harmonic content through the next voltage and current three-phase total demand distortion indices:

$$
\begin{aligned}
V T D D_{3 \phi} & =\sqrt{\frac{V_{e}^{2}-V_{e 1}^{2}}{V_{e}^{2}}} \\
I T D D_{3 \phi} & =\sqrt{\frac{I_{e}^{2}-I_{e 1}^{2}}{I_{e}^{2}}}
\end{aligned}
$$

where $V_{\mathrm{e} 1}$ and $\mathrm{I}_{\mathrm{e} 1}$ are the fundamental harmonic equivalent three-phase voltage and current, respectively.

According to their definition, the three-phase total demand distortion indices TDD measure the lines voltage and current waveforms no conformity respect to the sinusoidal waves. They are not affected by the unbalance conditions.

It is important to point out that these new indices may be calculated in a simple way from a commercial device harmonic measures. In fact, a power quality analyser supplies THD values, RMS values and fundamental component amplitude correspondent to each harmonic in each phase. So, the defined indices may be calculated for each measure in each phase. It makes easy to calculate the three-phase total distortion indices by mean of the next expressions:

$$
\begin{aligned}
V T D D_{3 \phi}= & \frac{1}{\sqrt{3}} \sqrt{V T H D_{L 1}^{2} \frac{V_{L 11}^{2}}{V_{e}^{2}}+V T H D_{L 2}^{2} \frac{V_{L 21}^{2}}{V_{e}^{2}}+} \\
& +V T H D_{L 3}^{2} \frac{V_{L 31}^{2}}{V_{e}^{2}} \\
I T D D_{3 \phi}= & \frac{1}{\sqrt{3}} \sqrt{I T H D_{L 1}^{2} \frac{I_{L 11}^{2}}{I_{e}^{2}}+I T H D_{L 2}^{2} \frac{I_{L 21}^{2}}{I_{e}^{2}}} \\
& +I T H D_{L 3}^{2} \frac{I_{L 31}^{2}}{I_{e}^{2}}++I T H D_{L 4}^{2} \frac{I_{L 41}^{2}}{I_{e}^{2}}
\end{aligned}
$$

These indices represent the root mean square $\mathrm{TDD}_{1 \phi}$ value, considering the neutral wire as the fourth phase.

To evaluate the unbalance conditions, it may be established a reference waveform, [10]. In this paper, it is defined a reference voltage waveform and a reference current waveform in an similar way: they are sinusoidal and positive sequence waveforms whose rms value are the equivalent three-phase voltage and current defined in (2) and (3), respectively.

From these references, it is defined a deviation index (DI) which measures the difference between the actual and the reference waveforms rms values. It is possible to calculate one DI equivalent to each phase by mean of the next expressions: 


$$
\begin{gathered}
D I_{1 \phi}=\sqrt{\frac{V_{L j}^{2}-V_{e}^{2}}{V_{e}^{2}}} \\
D I_{1 \phi}=\sqrt{\frac{I_{L j}^{2}-I_{e}^{2}}{I_{e}^{2}}}
\end{gathered}
$$

where $\mathrm{V}_{\mathrm{Lj}}$ and $\mathrm{I}_{\mathrm{Lj}}$ are the phase $\mathrm{j}$ voltage and current rms value.

The first phase DI index measure the difference between a sinusoidal positive sequence waveform whose rms value is the first phase rms value and the reference one. And so on the other phases.

The three-phase total unbalance indices (VTU and ITU) are defined as the root mean square of the DI indices as follows:

$$
\begin{gathered}
V T U=\frac{1}{\sqrt{3}} \sqrt{D I_{1}^{2}+D I_{2}^{2}+D I_{3}^{2}} \\
I T U=\frac{1}{\sqrt{3}} \sqrt{D I_{1}^{2}+D I_{2}^{2}+D I_{3}^{2}+D I_{4}^{2}}
\end{gathered}
$$

These indices estimate the waveforms no conformity respect to the reference one. In their calculation, only the measurements proposed by a commercial analyser have been taken into account.

However, the indices defined in (10), (11), (14) and (15) are not useful to make differences between the electric power poor quality responsibility: the supplier or the customer.

\section{Harmonic and unbalance registering in variable waveforms}

A measure equipment connection to register values each interval of time, shows that the voltage and current harmonics are variable along with the time due to the changes in the load conditions and in the system configuration. The study carried out in the second section is specially focused on the deterministic analysis, but it does not take into account this waveforms variability.

The figures 1 and 2 present the voltage and current THD variations in phase 1 , respectively, in a low voltage installation registering measures during a week each fifteen minutes. The installation corresponds to an University Complex composed by several buildings, some of them dedicated to personal offices and others to class-rooms.

This is the usual presentation from a network analyser software. The voltage THD has lower values during the weekend, when the consumption is lower than during the rest of the days. From Monday to Friday, it shows a cyclical behaviour. In the opposite, the current THD is higher during the weekend than during the rest of the days. In this case, the lower consumption makes to point out, in a higher way, the presence of distorting loads.

In any case, the THD variations usually happen in a fast way and in a random way.

Up now it has been usual to apply a deterministic study based on the worst case with the objective of supplying a security margin in the system design and operation. But it frequently goes to installation sizing higher than the necessary and to excessive costs. Consequently, it seems convenient for this harmonic content random behaviour to use statistical techniques that allow to quantify the distortion levels.

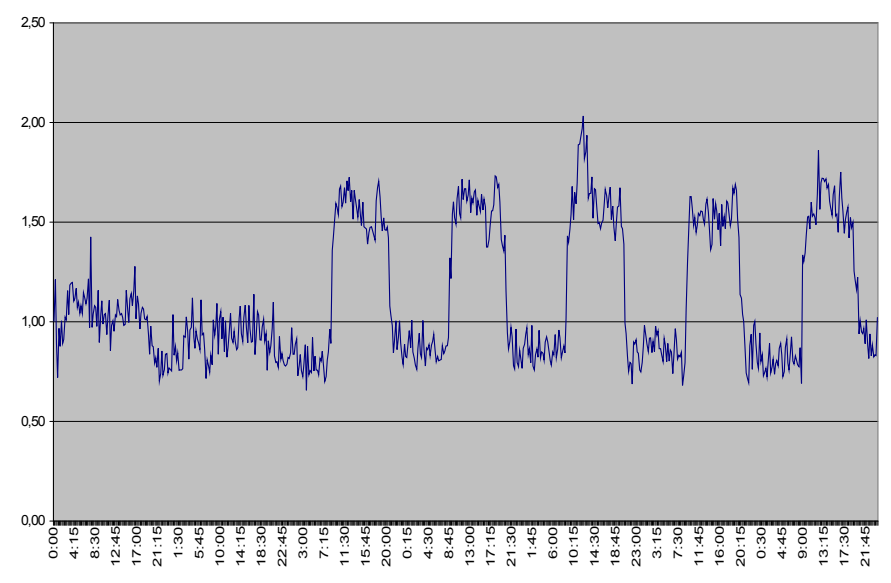

Figure 1. Phase 1 voltage THD evolution during a week.

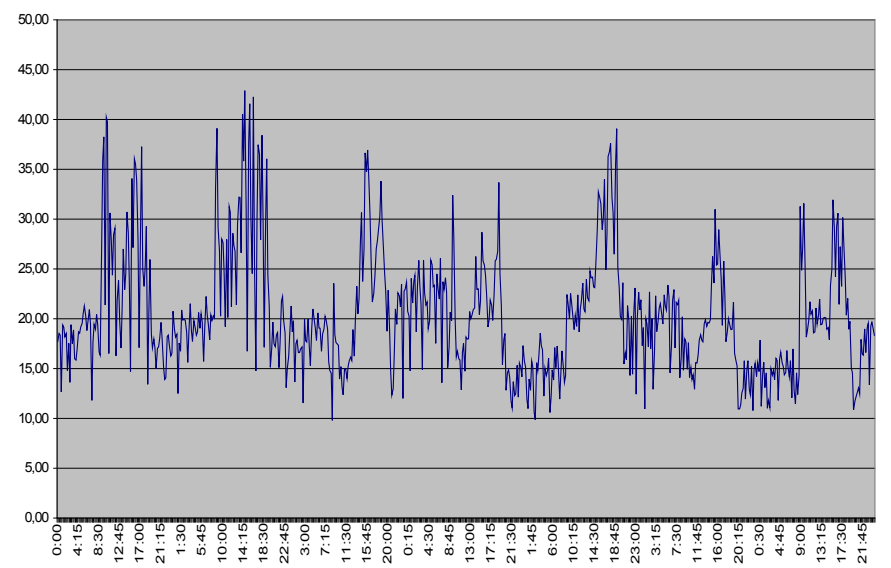

Figure 2. Phase 1 current THD evolution during a week.

\subsection{Measured data statistical characterization}

The industrial consumer may disposes of a great data base respect to an appropriate distortion index got from groups of measures during one day, one week or one month. This random group of data requires statistical techniques to be correctly treated.

Among the more adequate parameters and those used by the Probabilistic Aspect Task Force which belongs to the Harmonics Working Group from IEEE [12], they are included the minimum value, the maximum value, the average value and the typical deviation. The 
two last values constitute a centralization and dispersion measure, respectively, that allow to build a statistical distribution, that is a data group approximate image.

For a group of $\mathrm{n}$ measures $\mathrm{M}_{\mathrm{i}}, \mathrm{i}=1, \ldots, \mathrm{n}$, with a minimum value $M_{\operatorname{mim}}$ and a maximum value $M_{\max }$, the average value $M_{\text {avg }}$ and the standard deviation $\sigma_{M}$ are defined as follows:

$$
\begin{gathered}
M_{a v g}=\frac{\sum_{i=1}^{n} M_{i}}{n} \\
\sigma_{M}=\sqrt{\frac{\sum_{i=1}^{n}\left(M_{i}-M_{a v g}\right)^{2}}{n-1}}
\end{gathered}
$$

Alternatively, it is possible, from the distribution of $\mathrm{VTDD}_{3 \phi}$, ITDD $_{3 \phi}, \mathrm{VTU}$, ITU values, the 95 percentile, CP95, calculation. It presents the next characteristic: the 95\% of the index measures are lower than the Percentile 95. It is a more relevant parameter than the maximum value, because it is less sensitive to spore measures, [6]. In standards with distortion values limits fixed, the Percentile 95 is related to the fixed limits of distortion excessive values.

The statistical measure for the voltage and current registered data represented in figure 1 and 2 using the $\mathrm{TDD}_{3 \phi}$ indices are shown in table I. In the same way, table II presents the TU indices statistical parameters.

If the data are distributed as a normal or Gauss distribution, they are perfectly determined by the average and typical deviation values. The Gauss distribution assumption is more exact as the waveform random level increases and, besides, it depends on the fact that the signal comprises a deterministic component or not. If the signal is completely random, the Gauss distribution assumption is exact. In another way, if the statistical measures are obtained from a register which presents a relevant deterministic component, the actual probability distribution will go, in a relevant way, far from the Gauss distribution.

In the same way, if the data are distributed as a Rayleigh distribution, it is perfectly determined by the statistical parameters defined above.

Due to the usual difficulty to determine the best distribution to describe a data group, a more practical method consists of the absolute frequencies graphical representation. This kind of graphic, known as histogram, shows the occurrence of the total data group in different intervals. When they are scaled in a way that they cover an unity total area, the histograms represent the probability density function.

Figures 3 and 4 includes the voltage and current three-phase distortion indices histograms correspondent to the figures 1 and 2 data, respectively. The histograms present the data measured in a compact way although, in another way, the information about the particular instant that the different facts happened disappears.

Figure 3 shows that the $\operatorname{VTDD}_{3 \phi}$ distribution might be assumed as a Rayleigh distribution, being perfectly defined by the average and typical deviation values $(2,12$ and 0,52 , respectively).

On the other hand, figure 4 shows that the $\operatorname{ITDD}_{3 \phi}$ distribution should not be assumed as a normal one although both are similar. But taking into account its histogram form, it may be ensured that the probability of finding a value around the average one (30,73 A) is very similar to the correspondent to a Gauss distribution although that probability is higher for lower current values and lower for higher current values.

In any way, the figures 3 and 4 show, in a simple way, the values which happen with a highest frequency in the installation and the $M_{\text {avg }}$ and $\sigma_{\mathrm{M}}$ values correspond to the data group centralization and dispersion parameters.

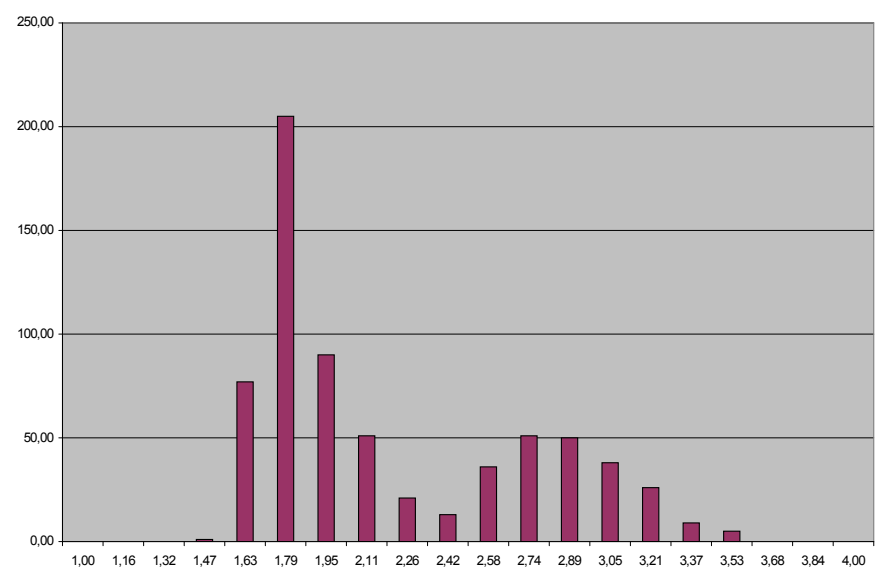

Figure 3. VTDD $3 \phi$ Histogram.

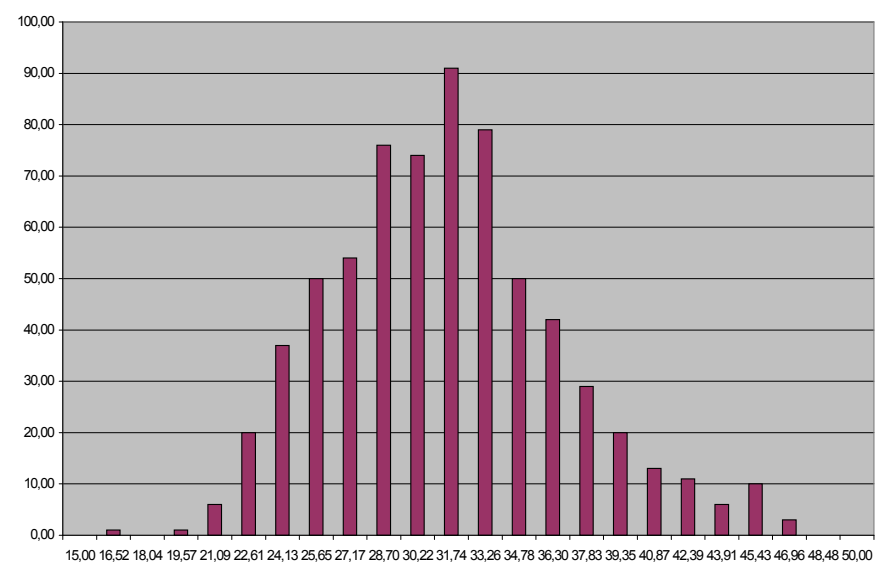

Figure 4. ITDD $3 \phi$ Histogram.

Taking into account the table 1 values, it may be pointed out the relation between the maximal value and the 95 percentile. For the $\operatorname{VTDD}_{3 \phi}$ the first one is 3,48 and the second one 3,08. The second one is a little lower because it is not affected by the spore values. In the case of ITDD $_{3 \phi}$ these values are 46,77 and 40,49. 
Figures 5 and 6 shows the VTU and the ITU indices histograms, respectively. It may be seen that none of them can be assumed as a Gauss, Ryleigh or any other reference distribution. Figure 5 shows a distribution centred and concentrated around its average value $(0,06$ $\mathrm{V}$ according to the table II). The typical deviation is not too high $(0,02$ according to the same table), what corroborates the concentration shown by the distribution.

Figure 6 shows a more disperse distribution (the typical deviation according to the table II is 0,07 ) centred but not concentrated around the average value $(0,76 \mathrm{~A})$. The distribution is wider in the left side than in the right one.

Looking at table II, which presents the statistical parameters relative to the TU indices, it can be seen that the 95 percentiles relative to the VTU and ITU are, respectively, 0,09 and 0,87 in front to the maximal values of 0,12 and 0,91 . Like in the case of total demand distortion indices, the current indices are higher than the voltage one.

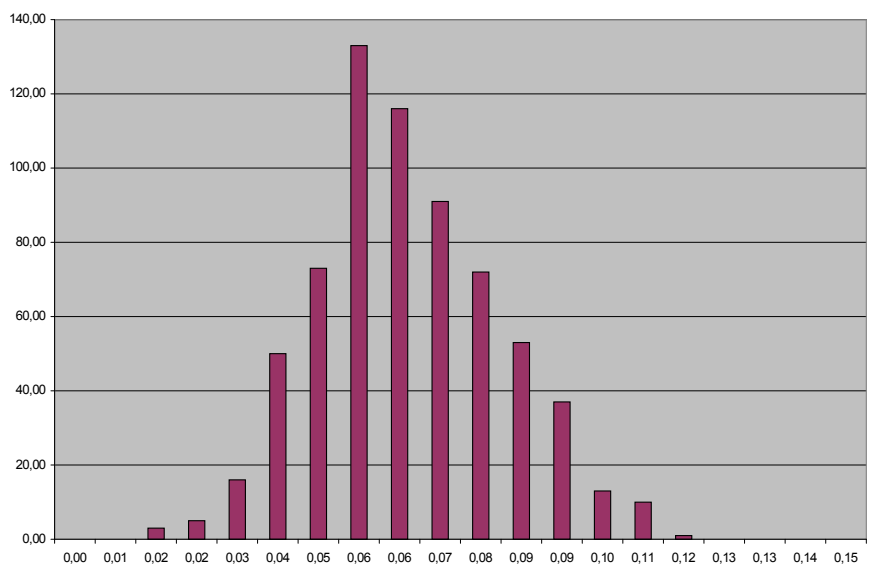

Figure 5. VTUD Histogram.

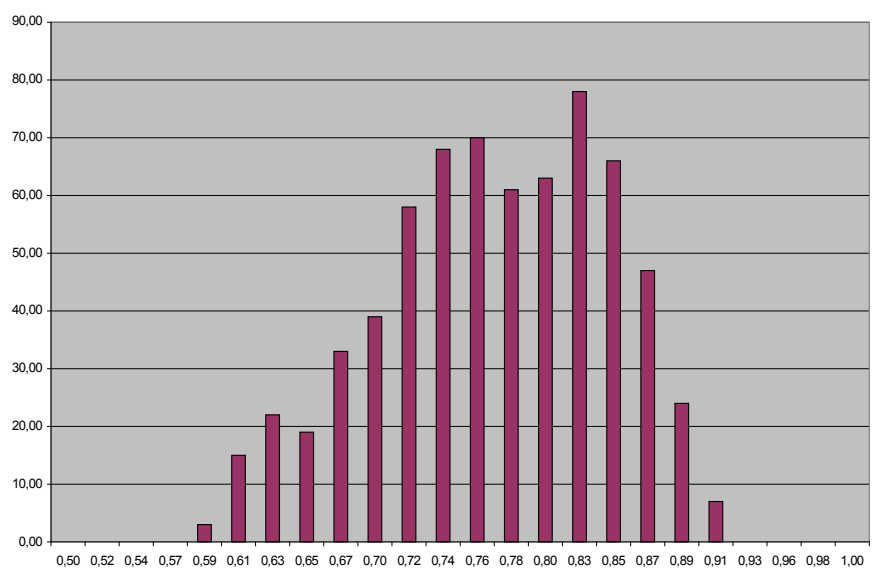

Figure 6. ITUD Histogram.

\section{Total average harmonic distortion and unbalance evaluation indices}

In this paper the voltage and current total demand distortion indices and the voltage and current total unbalance indices have been proposed. They are calculated as the correspondent to each phase root mean square values. But, usually, the three-phase power system is not perfectly balanced among each phase. So, the power consumed by each phase differs considerably from the other phases consumption. It affects in a relevant way to the current indices.

For that reason, it may be interesting to define a new distortion index which takes into account this new effect. So it is define the current total demand weighted distortion index $\left(\right.$ ITDDW $\left._{3 \phi}\right)$ as each phase ITDDW $_{1 \phi}$ indices root mean square. The single-phase total demand average distortion index is defined as the single-phase total demand distortion index pond rated by each phase apparent power, as follows:

$$
I T D D W_{1 \phi}=\frac{I T D D_{1 \phi} V A_{1 \phi}}{V A_{3 \phi}}
$$

where $\mathrm{VA}_{1 \phi}$ represents one phase apparent power and $\mathrm{VA}_{3 \phi}$ the three-phase apparent power defined as follows:

$$
V A_{3 \phi}=3 V_{e} I_{e}
$$

In this way, such phase contributes to the global index according to its relative importance.

These new definition may be applied to the THD index, too, obtaining the THDW $1 \phi$, as follows:

$$
I T H D W_{1 \phi}=\frac{I T H D_{1 \phi} V A_{1 \phi}}{V A_{3 \phi}}
$$

Appling this new indices to the expressions (10) and (11), the current three-phase total demand weighted distortion index may be defined as follows:

$$
\begin{aligned}
I T D D W_{3 \phi}= & \frac{1}{\sqrt{3}} \sqrt{I T H D W_{L 1}^{2} \frac{I_{L 11}^{2}}{I_{e}^{2}}+I T H D W_{L 2}^{2} \frac{I_{L 21}^{2}}{I_{e}^{2}}}+ \\
& +I T H D W_{L 3}^{2} \frac{I_{L 31}^{2}}{I_{e}^{2}}++I T H D W_{L 4}^{2} \frac{I_{L 41}^{2}}{I_{e}^{2}}
\end{aligned}
$$

Table III first row includes these indices values calculated in the reference network. The table III values show the three-phase index ITDDW $3 \phi$ where each phase contribution is weighted according to its consume importance. Its histogram is presented in the figure 7 . It must be pointed out that the distribution is a little similar to the Gauss distribution. It is centralized around the average value (17,74 $\mathrm{A}$ according to the table III first row). The distribution dispersion is not very high (the typical deviation value is 3,15 ).

On the other hand, table III first row show a 95 percentile of 23,53 in front of a higher maximal value of 27,87 .

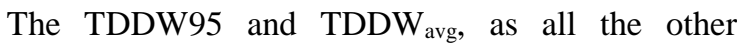
parameters, are calculated from a commercial analyser. 


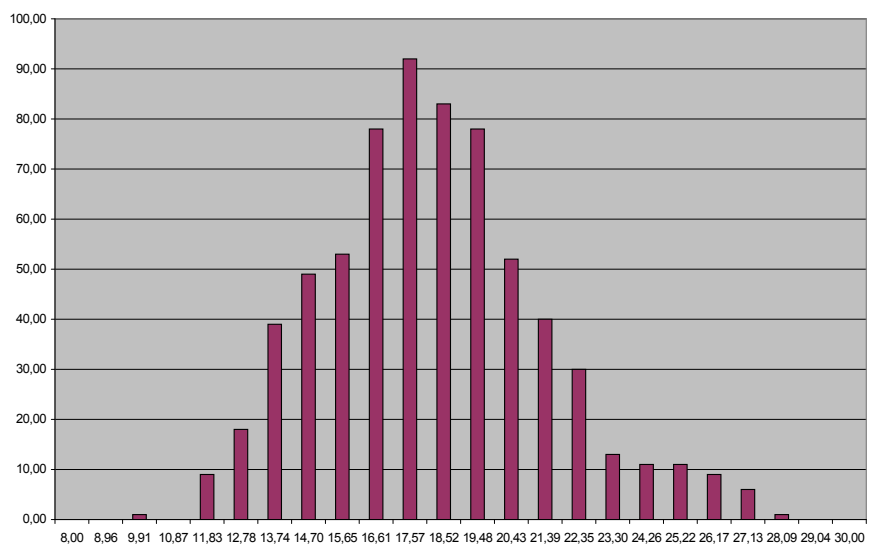

Figure 7. ITDDW $3 \phi$ Histogram.

In the same way ITU index may be weighted according to each phase apparent power to get the current weighted total unbalance index in the next way:

$$
I T U W=\frac{1}{\sqrt{3}} \sqrt{D I_{1}^{2} \frac{V A_{1}}{V A_{3 \phi}}+D I_{2}^{2} \frac{V A_{2}}{V A_{3 \phi}}++D I_{3}^{2} \frac{V A_{3}}{V A_{3 \phi}}+D I_{4}^{2}}
$$

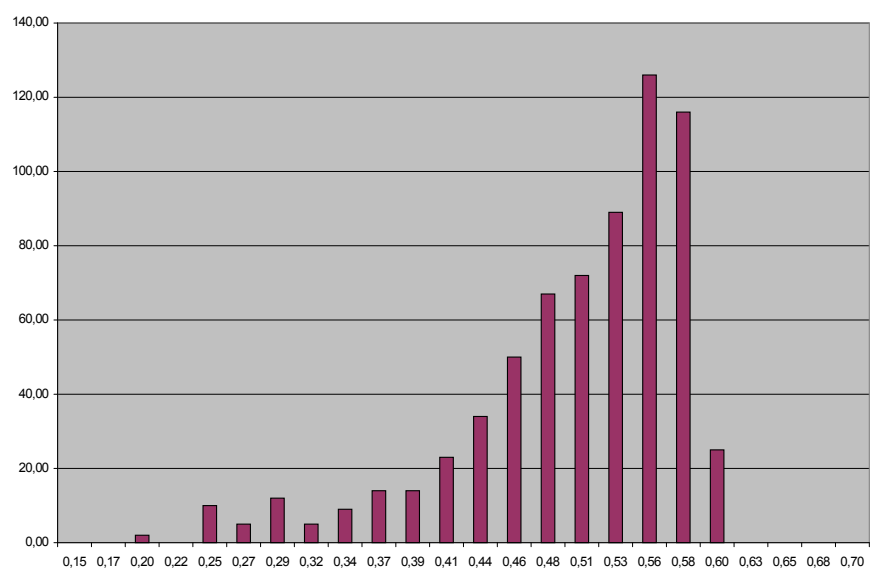

Figure 8. ITUDW Histogram.

Their statistical values are presented in table III second row and its histogram in the figure 8. As in the case of distortion indices, these new indices are lower than the not weighted. In spite of that, the typical deviation presented by the current weighted total unbalance index $(0,08)$ is higher than the same value correspondent to the same index without averaging. In fact, the dispersion presented by the weighted index histogram is higher than the equivalent to the not weighted one. The histogram correspondent to the weighted index is not so concentrated around the average value as the current total demand unbalance index whose histogram is presented in the figure 6 . In spite of that, the current total weighted unbalance index is centred around its average value $(0,49 \mathrm{~A})$. In any case, this histogram would not be assumed as a Gauss distribution.

The current total demand average unbalance index 95 percentile is 0,58 in front of its maximal value of 0,59 A.

\section{Conclusion}

In this paper they have been considered the possible problems associated to the electric power quality evaluation looking for voltage and current harmonic distortion and unbalance indices. Voltage and current are variable along with the time due to the non linear load performance conditions changes. It supposes a harmonic content measures random behaviour. In this paper they are presented several ways to descript statistically the data registered during the studied period. In particular, it is proposed a three-phase weighted total demand distortion index definition and a three-phase weighted total unbalance index which may be calculated from the measured registered from commercial analyzers. The presented distortion and unbalance evaluation indices are applied to recent measures got from a low voltage installation during a week.

\section{Acknowledgement}

This work is within the project "Harmonic Distortion Compensation in Electrical Installations using Different Configurations of Active Power Filters." DPI2003-01336, financed by the CICYT (Ministerio de Ciencia y Tecnología, Spain).

\section{References}

[1] Task Force on Probabilistic Aspects of Harmonics, 1998, "Time-varying harmonics: Part I-Characterizing measured data", IEEE Trans. on Power Delivery, Vol. 13, No 3

[2] Task Force on Probabilistic Aspects of Harmonics, 2002, "Time-varying harmonics: Part II-Harmonic summation and propagation", IEEE Trans. on Power Systems, Vol. 17, No 1

[3] R. Sasdelli, G. Del Gobbo, G. Iuculano, 2000 "Quality management for electricity as a processed material". IEEE Trans. on Instrumentation and Measurement, Vol. 49, No. 2.

[4] G.T. Heydt, W.T. Jewel, 1998 "Pitfall of electric power quality indices", IEEE Trans. on Power Delivery, Vol.13, No.2. [5] G.T. Heydt, E. Gunther, 1996 "Post-measurement processing of electric power quality data", IEEE Trans. on Power Delivery, Vol. 11, No. 4.

[6] D.D. Sabin, D.L. Brooks, A. Sundaram, 1999, "Indices for assessing harmonic distortion from power quality measurements: definitions and benchmark data", IEEE Trans. on Power Delivery, Vol. 14, No. 2

[7] M.S. Kandil, S.A. Farghal, A. Elmitwally, "Refined Power Quality Indices", IEE Proc-Gener. Transm. Distrib., Vol. 148, No. 6, Nov 2001.

[8] E.F. Fuchs, D.J. Roesler, H.A.S. Masoum, "Are Harmonic Recommendations According to IEEE and IEC Too Restrictive?", IEEE Transactions on Poer Delivery, Vol. 19, No. 4, October 2004.

[9] A.E. Emanuel, "Summary of IEEE Standard 1459: Definitions for the Measurement of Electric Power Quantities Under Sinusoidal, Nonsinusoidal, Balanced, or Unbalanced Conductions", IEEE Trans. On Ind. Appl., Vol. 40, No. 3, 2004, pp.869-876.

[10] A. Dell'Aquila, M. Marinelli, V.G. Monopoli, P.Zanchetta, "New Power-Quality Assessment Criteria for Suplí Systems Ander Unbalanced and Nonsinusoidal Conditions", IEEE Transactions onPower Delivery, Vol. 19, No. 3, July 2004. 
Table I. Statistical values to evaluate harmonic distortion

\begin{tabular}{|c|c|c|c|c|c|}
\hline & Max & Min & Avg & Desv & CP95 \\
\hline$\overline{\text { VTDD }_{3 \phi}}$ & 3,48 & 1,47 & 2,12 & 0,52 & 3,08 \\
\hline$\overline{\text { ITDD }_{3 \phi}}$ & 46,77 & 16,51 & 30,73 & 5,18 & 40,49 \\
\hline
\end{tabular}

Table II. Statistical values to evaluate unbalance

\begin{tabular}{|c|c|c|c|c|c|}
\hline & Max & Min & Avg & Desv & CP95 \\
\hline VTU & 0,12 & 0,01 & 0,06 & 0,02 & 0,09 \\
\hline ITU & 0.91 & 0,58 & 0,76 & 0,07 & 0,87 \\
\hline
\end{tabular}

Table III. Statistical values to evaluate current weighted indices

\begin{tabular}{|c|c|c|c|c|c|}
\hline & $\begin{array}{l}\text { Max } \\
\end{array}$ & Min & Avg & 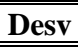 & CP95 \\
\hline ITDDW $_{3 \phi}$ & 27,87 & 9,34 & 17,74 & 3,15 & 23,57 \\
\hline ITUW & 0,59 & 0,19 & 0,49 & 0,08 & 0,58 \\
\hline
\end{tabular}

